\title{
MicroRNA-10b inhibits proliferation, migration and invasion in cervical cancer cells via direct targeting of insulin-like growth factor-1 receptor
}

\author{
REN HOU ${ }^{1}$, DAIXIAN WANG ${ }^{2}$ and JIAN LU ${ }^{3}$ \\ ${ }^{1}$ Department of Gynecology, Affiliated Hospital of Guilin Medical University, Guilin, Guangxi 541001; \\ ${ }^{2}$ Department of Orthopedics, The People's Hospital of Rizhao, Rizhao, Shandong 276826; ${ }^{3}$ Department of Orthopedics, \\ The Affiliated Hospital of Guilin Medical University, Guilin, Guangxi 541001, P.R. China
}

Received February 12, 2016; Accepted February 3, 2017

DOI: $10.3892 / \mathrm{ol} .2017 .6033$

\begin{abstract}
MicroRNAs are deregulated in numerous types of human cancers and have crucial roles in the carcinogenesis and progression of human cancers. MicroRNA-10b (miR-10b) has been studied in several types of human cancer. However, the expression and roles of $\mathrm{miR}-10 \mathrm{~b}$ in cervical cancer remain unknown. In the present study, the expression, functions and molecular mechanisms of miR-10b were explored in cervical cancer. The present data revealed that miR-10b was significantly downregulated in cervical cancer tissues and cell lines. In addition, miR-10b overexpression inhibited the proliferation, migration and invasion of cervical cancer cells, while miR-10b under-expression had the opposite effect. Based on bioinformatics analysis, a luciferase reporter assay and western blot analysis, insulin-like growth factor-1 receptor (IGF-1R) was identified as a direct target of miR-10b in cervical cancer. In addition, IGF-1R small interfering RNA-mediated knockdown of IGF-1R also inhibited the proliferation, migration and invasion of the cervical cancer cells. In conclusion, the present study demonstrated that miR-10b serves an important role in cervical cancer progression by targeting IGF-1R.
\end{abstract}

\section{Introduction}

Cervical cancer is the third most commonly diagnosed malignancy and the fourth leading cause of cancer-associated mortality in women worldwide, with $\sim 530,000$ new cases and 275,100 cervical cancer-associated mortalities occurring in females in 2008 (1). The initiation and progression of cervical

Correspondence to: Professor Jian Lu, Department of Orthopedics, The Affiliated Hospital of Guilin Medical University, 15 Lequn Road, Guilin, Guangxi 541001, P.R. China

E-mail: glmu_jianlu@163.com

Key words: microRNA-10b, cervical cancer, insulin-like growth factor-1 receptor, proliferation, migration, invasion cancer is a multi-step process, involving multiple factors and the transformation of normal cervical epithelium into cervical intraepithelial neoplasia, which subsequently transforms into invasive cervical cancer $(2,3)$. A number of studies have demonstrated that persistent infection with high-risk human papillomavirus (HPV) serves an important role in the initiation and progression of cervical cancer (4-6). However, previous studies have shown that HPV infection alone is insufficient to induce malignant changes and that other factors must contribute to cervical carcinogenesis and progression $(7,8)$. Accumulated studies reported that abnormal expression or activity of specific genes is responsible for the pathogenesis of cervical cancers (9-11). Currently, surgery, radiotherapy and chemotherapy are the main therapies for patients with cervical cancer (12). However, subsequent to these treatments, $30 \%$ of patients developed cancer recurrence, lymph node recurrence or distant metastasis and eventually obtained an unfavorable prognosis (13). Therefore, it is of great significance to fully understand the molecular mechanisms underlying the biology, genetics, causes and cellular origin of cervical cancer, which are important for developing novel therapeutic strategies for patients with cervical cancer.

MicroRNAs (miRNAs) are a sizable group of endogenous, non-protein-coding and short RNAs of 21-23 nucleotides, which negatively regulate their target mRNAs through binding the 3' untranslated regions (3'UTRs) of mRNAs, causing mRNA degradation or inhibiting translation $(14,15)$. miRNAs regulate the activity of $>30 \%$ of human genes, and therefore perform important roles in a variety of physiological and pathological processes, including cell proliferation, apoptosis, metastasis, glucose and lipid metabolism, and infection and immune responses (16-20). Previous studies have demonstrated that miRNAs are deregulated in numerous types of human cancers, and are associated with tumorigenesis and development by regulation of oncogenes or tumor suppressors (21-23). These previous findings indicated the important functions of miRNAs in the initiation and progression of human cancers, and demonstrate the potential of miRNAs as efficient therapeutic targets for cancer treatment.

In the present study, miR-10b was identified as a tumor suppressor miRNA in cervical cancer. The present data 
revealed that miR-10b was significantly downregulated in cervical cancer tissues and cell lines. It was also revealed that miR-10b overexpression inhibited cervical cancer cell proliferation, migration and invasion, while miR-10b under-expression had the opposite effect. Furthermore, the present study demonstrated that insulin-like growth factor-1 receptor (IGF-1R) was directly regulated by $\mathrm{miR}-10 \mathrm{~b}$ in cervical cancer, and subsequent downregulation of IGF-1R mimicked the inhibitory effects of miR-10b on cervical cancer.

\section{Materials and methods}

Tumor specimens. The present study was approved by the Ethics Committee of the Affiliated Hospital of Guilin Medical University (Guangxi, China). A total of 46 cases of cervical cancer tissues and adjacent normal cervical epithelial tissues were collected from patients who were newly diagnosed with cervical cancer between March 2012 and August 2014 in the Affiliated Hospital of Guilin Medical University. No radiotherapy or chemotherapy was performed in any patients prior to surgery. Fresh tissues were stored in liquid nitrogen prior to RNA extraction.

Cell culture and transfection. A total of 5 human cervical cancer cell lines (HeLa, CaSki, HT-3, C-33A and SiHa), a normal human cervix epithelial cell line (Ect1/E6E7) and the HEK293T cell line were all purchased from American Type Culture Collection (Manassas, VA, USA). All cell lines were cultured in Dulbecco's modified Eagle's medium (DMEM; Thermo Fisher Scientific, Inc., Waltham, MA, USA) containing $10 \%$ fetal bovine serum (FBS; Thermo Fisher Scientific, Inc.) and $1 \%$ antibiotic/antimycotic (Thermo Fisher Scientific, Inc.) in a humidified air atmosphere of $5 \% \mathrm{CO}_{2}$ at $37^{\circ} \mathrm{C}$.

The miR-10b mimics, corresponding negative controls (NC), miR-10b inhibitor, NC inhibitor and luciferase report vectors [pGL3-IGF-1R-3'UTR wild type (Wt) and pGL3-IGF-1R-3'UTR mutant (Mut)] were obtained from GenePharma (Shanghai, China). IGF-1R small interfering RNA (siRNA) and NC siRNA were chemically synthesized by Guangzhou RiboBio Co., Ltd. (Guangzhou, China). Transfection of mimics, inhibitors, siRNAs and luciferase report vectors was performed using Lipofectamine ${ }^{\circledR} 2000$ (Invitrogen; Thermo Fisher Scientific, Inc.), according to the manufacturer's protocol.

RNA extraction and reverse transcription-polymerase chain reaction $(R T-P C R)$. Total RNA was isolated from tissues and cells using the mirVana miRNA isolation kit (Ambion; Thermo Fisher Scientific, Inc.), according to the manufacturer's protocol. Reverse transcription was performed with the PrimeScript ${ }^{\mathrm{TM}}$ RT reagent kit (Takara Biotechnology Co. Ltd., Dalian, China), followed by RT-PCR conducted using SYBR $^{\circledR}$ Premix Ex Taq ${ }^{\mathrm{TM}}$ II (Takara Biotechnology Co., Ltd.). The expression level of miR-10b was normalized to the expression level of U6 small nuclear RNA. U6 small nuclear RNA was used as a loading control. All reactions were performed on the Applied Biosystems 7500 real-time PCR system (Thermo Fisher Scientific, Inc.) and run in triplicate.

Cell proliferation assay. Cervical cancer cells $\left(3 \times 10^{3}\right.$ cells $\left./ 100 \mu \mathrm{l}\right)$ were seeded onto 96-well plates. Following incubation for
6-8 h, cells were transfected with mimics, inhibitor or siRNAs and cultured at $37^{\circ} \mathrm{C}$ for $24,48,72$ and $96 \mathrm{~h}$. Cellular proliferation was measured using MTT assay (Sigma Chemical Co., St Louis, MO, USA). Briefly, $20 \mu \mathrm{l}$ MTT solution ( $5 \mathrm{mg} / \mathrm{ml}$ ) was added to each well and incubated at $37^{\circ} \mathrm{C}$ for an additional $4 \mathrm{~h}$. Culture medium was then removed and formazan crystals were dissolved in dimethyl sulfoxide. The optical density (OD) at $490 \mathrm{~nm}$ was detected with an enzyme linked immunosorbent assay reader (Dasit Group S.p.A, Milan, Italy).

Migration and invasion assay. Migration and invasion assays were performed using Transwell chambers (pore size, $8 \mu \mathrm{m}$; Costar; Corning Incorporated, Corning, NY, USA). For the migration assay, $5 \times 10^{4}$ cells in $100 \mu \mathrm{l}$ of FBS-free culture medium were added to the upper chambers, and $500 \mu \mathrm{l}$ of DMEM medium supplemented with $20 \%$ FBS was added to the lower chambers as a chemoattractant. Following incubation at $37^{\circ} \mathrm{C}$ for $24 \mathrm{~h}$, cells remaining on the upper membrane of the Transwell chamber were removed using a cotton swab. The migrated cells were fixed, stained at room temperature with $0.1 \%$ crystal violet, washed and then dried in air. For the invasion assay, $5 \times 10^{4}$ cells in $100 \mu \mathrm{l}$ of FBS-free culture medium were added to the upper chambers, which were pre-coated with Matrigel (BD Biosciences, San Jose, CA, USA). The subsequent steps were similar to the migration assay, but the Transwell chambers were incubated at $37^{\circ} \mathrm{C}$ for $48 \mathrm{~h}$. Images of five randomly selected fields of the migrated/ invaded cells were captured and the cells were counted, under an inverted microscope (magnification, x200; CKX41; Olympus Corporation, Tokyo, Japan).

Bioinformatics analysis and luciferase report assay. To predict the potential targets of miR-10b, bioinformatics analysis was conducted using microRNA.org (http://www.microrna.org/ microrna/) and TargetScan (http://www.targetscan.org/).

HEK293T cells were seeded onto 24-well plates, and were co-transfected with pGL3-IGF-1R-3'UTR Wt or pGL3-IGF-1R-3'UTR Mut, and miR-10b mimics or NC. The transfected cells were cultured for $48 \mathrm{~h}$, and luciferase activities were detected using the Dual-Luciferase ${ }^{\circledR}$ reporter assay system (Promega Corporation, Madison, WI, USA), according to the manufacturer's protocol. Renilla luciferase activity was normalized to Firefly luciferase activity. Results were obtained from three independent experiments.

Western blot analysis. Cells were washed twice with cold PBS (Thermo Fisher Scientific, Inc.) and lysed with radioimmunoprecipitation assay lysis buffer. Equal amounts of protein were dissolved in $10 \%$ SDS-PAGE and blotted onto polyvinylidene difluoride membranes (EMD Millipore, Billerica, MA, USA). The membranes were then blocked with $5 \%$ non-fat milk in TBS with Tween-20 (TBST), followed by incubation with the following primary antibodies: Mouse anti-human monoclonal IGF-1R (dilution, 1:1,000; catalog no. sc-81464; Santa Cruz Biotechnology, Inc., Dallas, TX, USA) and mouse anti-human monoclonal GADPH (dilution, 1:1,000; catalog no. sc-59540; Santa Cruz Biotechnology, Inc.). Following incubation overnight at $4^{\circ} \mathrm{C}$, the membranes were washed three times with TBST, incubated with goat anti-mouse horseradish peroxidase-conjugated secondary antibodies (dilution, 1:3,000; 

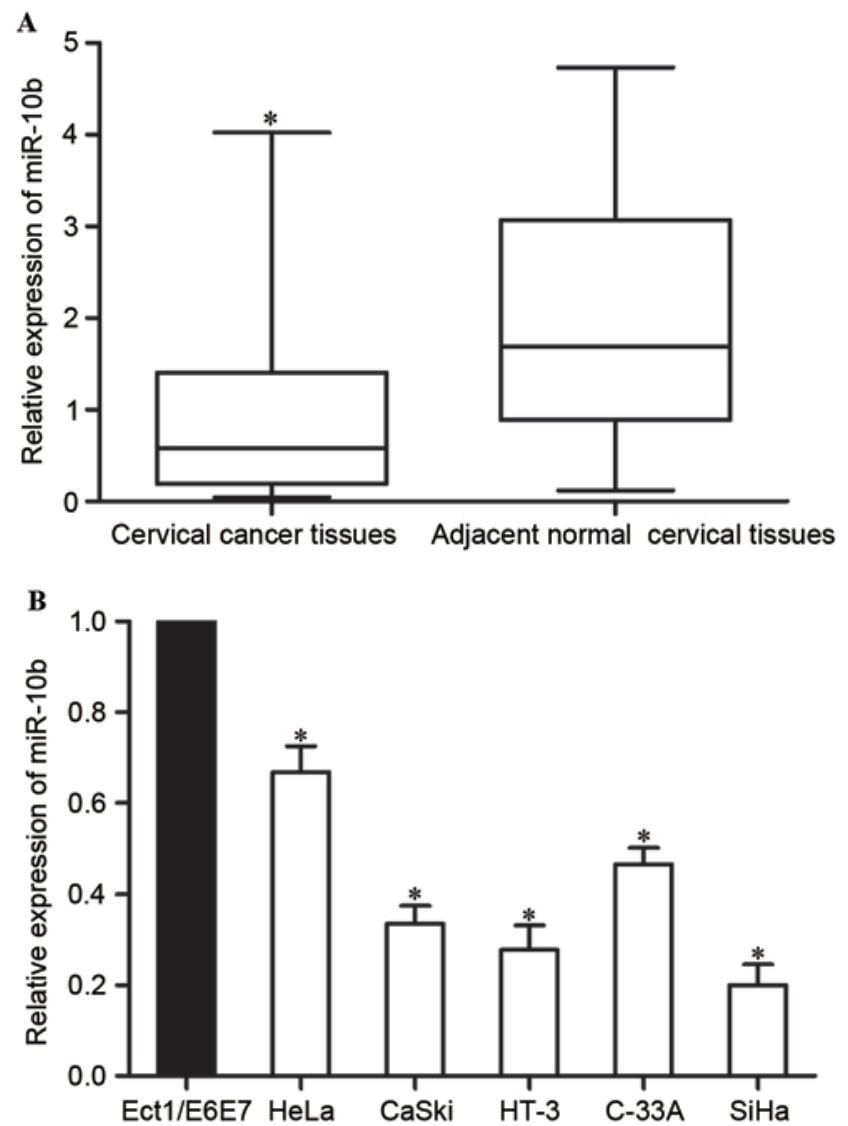

Figure 1. Low expression level of miR-10b in cervical cancer. (A) miR-10b expression levels in 46 paired cervical cancer tissues and adjacent normal cervical epithelial tissues. (B) miR-10b expression levels in five cervical cancer cell lines and normal human cervix epithelial Ect1/E6E7 cell line. ${ }^{*} \mathrm{P}<0.05$. miR-10b, microRNA-10b.

catalog no. sc-2005; Santa Cruz Biotechnology, Inc.) and visualized by enhanced chemiluminescence (EMD Millipore), according to the manufacturers protocol. GADPH was used as a loading control.

Statistical analysis. All values were presented as the mean \pm standard deviation. Differences between groups were assessed using SPSS version 13.0 software (SPSS, Inc., Chicago, IL, USA). P $<0.05$ was considered to indicate a statistically significant difference.

\section{Results}

miR-10b is downregulated in cervical cancer. As first step of the present study, the expression levels of miR-10b in cervical cancer tissues and adjacent normal cervical epithelial tissues were measured. The results revealed that miR-10b expression was significantly lower in cervical cancer tissues compared with adjacent normal cervical epithelial tissues, indicating that miR-10b may act as a tumor suppressor in cervical cancer $(\mathrm{P}<0.05$; Fig. 1A).

The expression of miR-10b was then examined in five human cervical cancer cell lines (HeLa, CaSki, HT-3, C-33A and $\mathrm{SiHa}$ ). The results revealed that miR-106 was lower in all five examined cell lines, compared with the normal human cervix epithelial Ect1/E6E7 cell line ( $\mathrm{P}<0.05$; Fig. 1B).
miR-10b negatively regulates the proliferation, migration and invasion of cervical cancer cells. The expression of miR-10b in HeLa cells was the highest in the five cell lines, while $\mathrm{SiHa}$ had the lowest miR-10b expression levels. Considering the present results, HeLa and SiHa cells were selected for subsequent experiments. SiHa cells were transfected with miR-10b mimics or NC, and HeLa cells were transfected with miR-10b inhibitor or NC inhibitor. At $48 \mathrm{~h}$ following transfection, the transfection efficiency was measured by RT-PCR. The results revealed that the miR-10b mimic significantly upregulated expression levels in $\mathrm{SiHa}$ cells, and the miR-10b inhibitor downregulated miR-10b expression in HeLa cells $(\mathrm{P}<0.05$; Fig. 2A).

To investigate the effect of miR-10b on cervical cancer cell proliferation, cell proliferation assays (MTT assays) were performed. Compared with the control groups, miR-10b mimics inhibited the proliferation of SiHa cells, while the miR-10b inhibitor prompted proliferation of $\mathrm{HeLa}$ cells $(\mathrm{P}<0.05$; Fig. 2B). Migration and invasion assays were performed to explore the effects of miR-10b on metastasis of cervical cancer cells. As shown in Fig. 2C, the miR-10b mimic reduced SiHa cell migration and invasion abilities $(\mathrm{P}<0.05)$. HeLa cell migration and invasion abilities were increased following transfection with miR-10b inhibitor compared with that in cells transfected with $\mathrm{NC}$ inhibitor $(\mathrm{P}<0.05)$. These results indicated that miR-10b may act as a tumor suppressor in cervical cancer.

$I G F-1 R$ is a direct target of miR-10b in cervical cancer. It is generally accepted that miRNAs exert their functions through binding to the 3'UTR of target mRNAs and regulating their expression. Therefore, bioinformatics analysis was performed with microRNA.org and TargetScan. As shown in Fig. 3A, IGF-1R was predicated to be a potential target of miR-10b.

To investigate whether IGF-1R was a genuine target of miR-10b, luciferase report assay was conducted. pGL3-IGF-1R-3'UTR Wt or pGL3-IGF-1R-3'UTR Mut, along with miR-10b mimics or NC, were co-transfected into HEK293T cells. As shown in Fig. 3B, the luciferase activities of pGL3-IGF-1R-3'UTR Wt were significantly suppressed when miR-10b mimics were co-transfected $(\mathrm{P}<0.05)$. By contrast, the luciferase activities of the pGL3-IGF-1R-3'UTR Mut were unaffected by transfection of miR-10b mimics ( $P>0.05)$. These results indicated that miR-10b directly targeted the 3'UTR of IGF-1R.

The effect of miR-10b on the expression levels of IGF-1R was also measured. The results revealed that miR-10b mimics decreased IGF-1R expression in SiHa cells, while miR-10b inhibitor improved IGF-1R levels in HeLa cells $(\mathrm{P}<0.05)$. IGF-1R was a direct target of miR-10b in cervical cancer.

$I G F-1 R$ is involved in miR-10b-mediated proliferation, migration and invasion of cervical cancer cells. To evaluate whether IGF-1R was involved in miR-10b-mediated proliferation, migration and invasion of cervical cancer cells, IGF-1R siRNA was adopted to reduce IGF-1R expression. At $72 \mathrm{~h}$ following transfection, western blot analysis was performed to assess its transfection efficiency. As shown in Fig. 4A, 

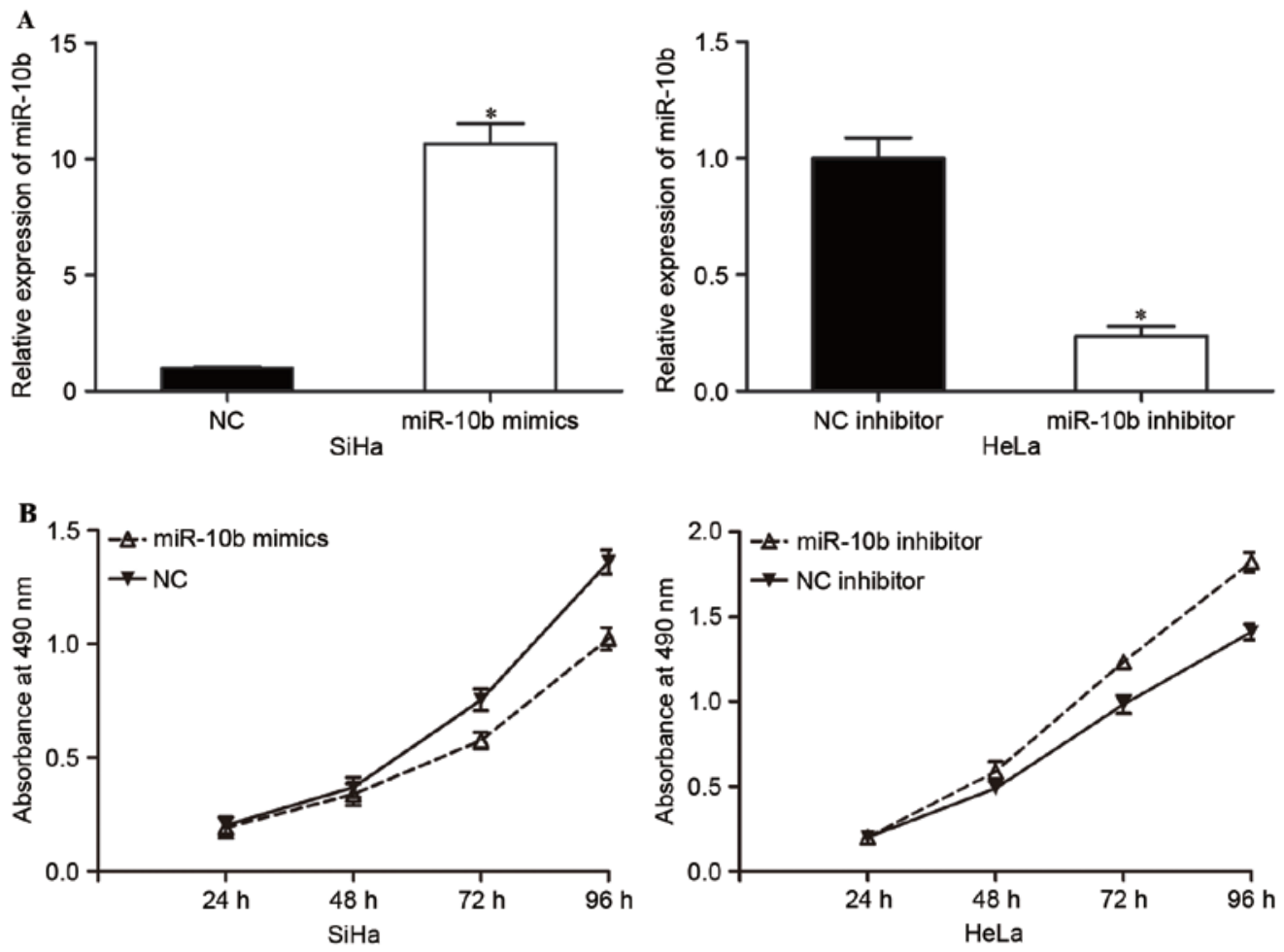

C miR-10b mimics NC
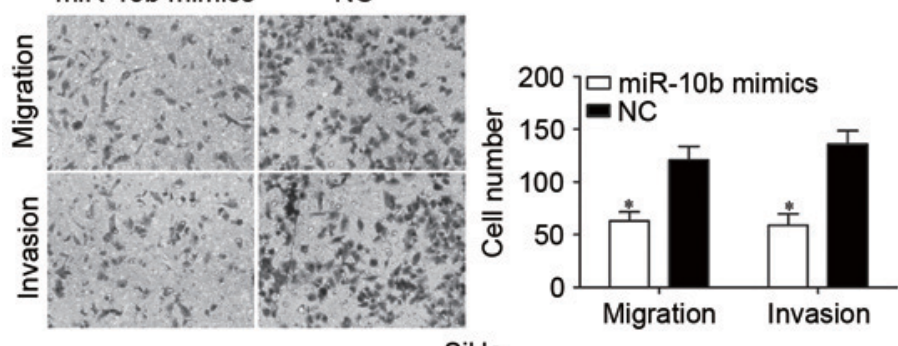

miR-10b inhibitor NC inhibitor

$\mathrm{SiHa}$
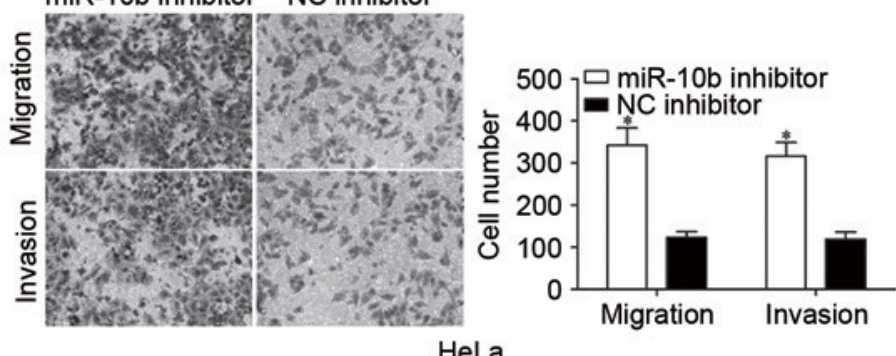

Figure 2. Effects of miR-10b on proliferation, migration and invasion of cervical cancer cells. (A) Expression levels of miR-10b in SiHa cells transfected with miR-10b mimics or NC, and HeLa cells transfected with miR-10b inhibitor or NC inhibitor. (B) miR-10b mimics inhibited proliferation of SiHa cells, and miR-10b inhibitor prompted proliferation of HeLa cells. (C) Migration and invasion assays demonstrated that numbers of migrated and invaded SiHa cells transfected with miR-10b mimic were lower compared with cells transfected with NC. Numbers of migrated and invaded HeLa cells transfected with miR-10b inhibitor were higher than cells transfected with NC inhibitor (magnification, x200). "P<0.05. miR-10b, microRNA-10b; NC, negative control.

IGF-1R siRNA significantly decreased IGF-1R expression in $\mathrm{SiHa}$ cells when compared with cells transfected with $\mathrm{NC}$ siRNA $(\mathrm{P}<0.05)$.

Subsequently, cell proliferation, migration and invasion assays were performed to investigate the effects of IGF-1R siRNA on cell proliferation, migration and invasion. The present data revealed that IGF-1R significantly inhibited proliferation $(\mathrm{P}<0.05$; Fig. 4B), migration $(\mathrm{P}<0.05$; Fig. $4 \mathrm{C})$ and invasion $(\mathrm{P}<0.05$; Fig. $4 \mathrm{C})$ of cervical cancer cells, indicating that IGF-1R acted as a downstream effector in the miR-10b-mediated proliferation, migration and invasion of cervical cancer cells.

\section{Discussion}

The main factors affecting the clinical prognosis of metastatic and recurrent cervical cancer have not been fully investigated. It is important to explore the molecular mechanisms underlying 
A

IGF-1R 3' UTR Wt 5' ...CUCUGUGCGAAUCCCCAGGGUAA...3' hsa-miR-10b 3' GUGUUUAGCCAAGUGUCCCAU 5' IGF-1R 3' UTR mut 5' ...CUCUGUGCGAAUCCCUGUCCCAA... 3'
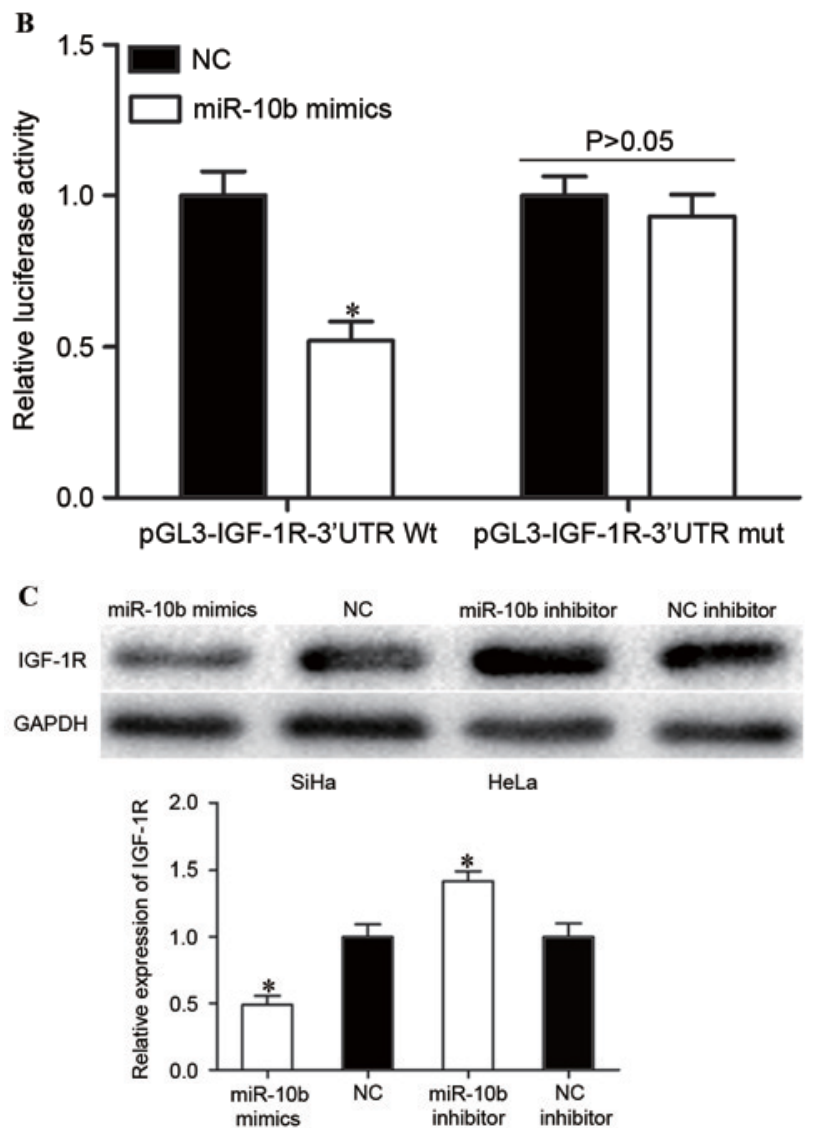

Figure 3. IGF-1R is a direct target of miR-10b in cervical cancer. (A) Predicted miR-10b target sequence in the 3'UTR of IGF-1R and positions of mutated nucleotides in the 3'UTR of IGF-1R. (B) HEK293T cells were transfected with luciferase report vectors, together with miR-10b mimics or NC. Luciferase activities were measured at $48 \mathrm{~h}$ post-transfection. (C) The protein expression levels of IGF-1R were determined by western blot analysis. "P<0.05. IGF-1R, insulin-like growth factor-1 receptor; miR-10b, microRNA-10b; UTR, untranslated region; Wt, wild type; mut, mutant; NC, negative control.

the initiation and development of cervical cancer. A growing amount of evidence has demonstrated that miRNAs are important regulators of various types of biological processes in cancers, including cell proliferation, cell cycle, apoptosis, invasion and migration (24-26). Furthermore, the abnormal expression of miRNAs is associated with carcinogenesis and progression of cancer (27-29). Therefore, investigating the expression and functions of miRNAs in cervical cancer may benefit the development of improved strategies for refractory cervical cancer treatment.

The present data revealed that expression levels of miR-10b were lower in cervical cancer tissues compared with adjacent normal cervical epithelial tissues. Consistently, five cervical cancer cell lines also expressed lower miR-10b. In functional studies, miR-10b overexpression significantly inhibited proliferation, migration and invasion of cervical cancer cells, while miR-10b under-expression had the opposite effects. To the best of our knowledge, it was demonstrated for the first time that IGF-1R was a direct target of miR-10b in cervical
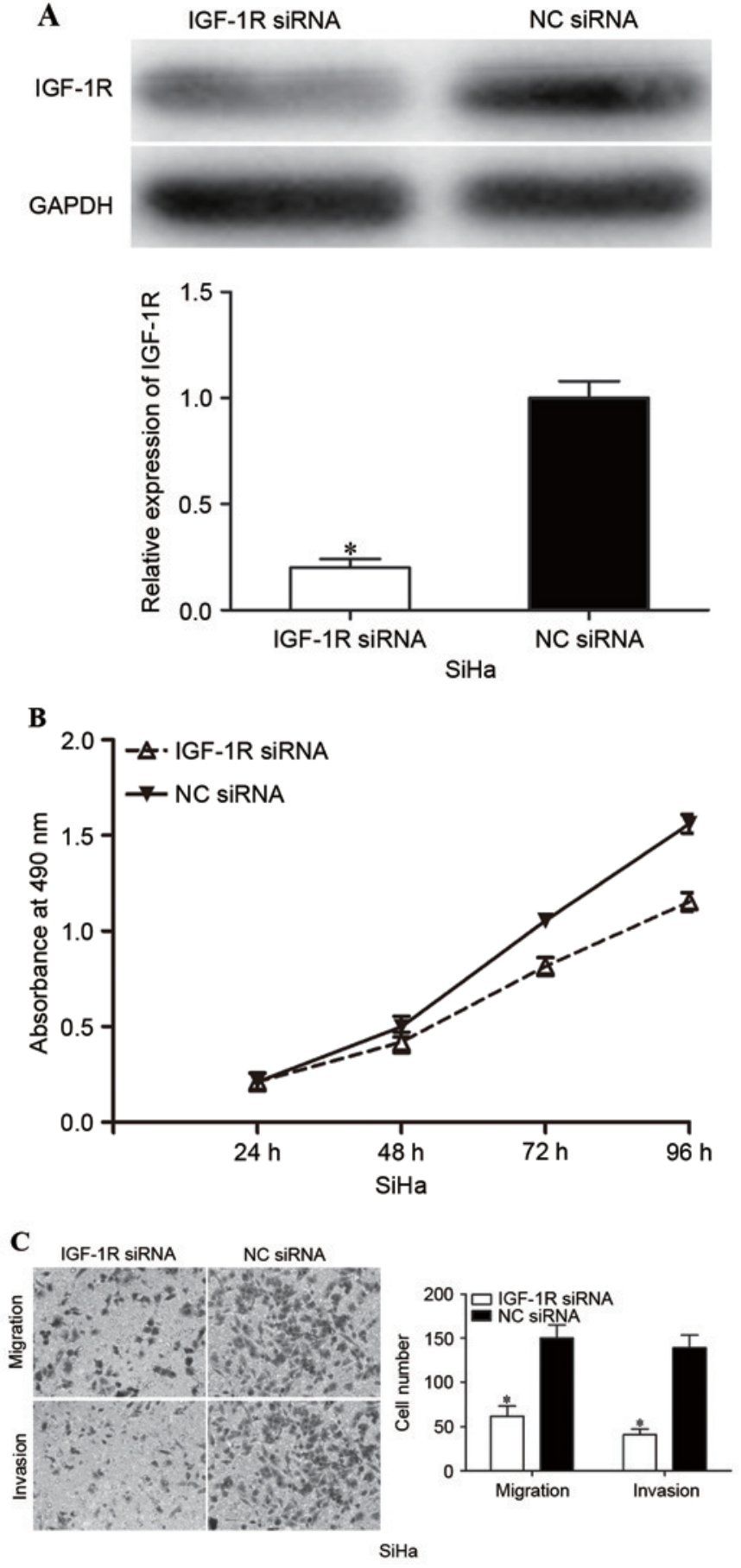

Figure 4. IGF-1R siRNA inhibited proliferation, migration and invasion of cervical cancer cells. (A) Western blot analysis was performed to detect IGF-1R expression. (B) IGF-1R siRNA inhibited proliferation of SiHa cells. (C) IGF-1R siRNA inhibited migration and invasion of SiHa cells (magnification, $\mathrm{x} 200)$. ${ }^{\mathrm{P}}<0.05$. IGF-1R, insulin-like growth factor-1 receptor; siRNA, small interfering RNA; NC, negative control.

cancer. Thus, the present findings indicated that in cervical cancer, miR-10b may act as a tumor suppressor miRNA that is commonly downregulated in cancer tissues, and its overexpression may inhibit cervical cancer growth and metastasis.

Previously, miR-10b was revealed to be upregulated in numerous types of human cancers, including melanoma (30), gastric cancer (31), non-small-cell lung cancer (32), glioma (33), colorectal cancer (34), bladder cancer (35), breast cancer (36), nasopharyngeal carcinoma (37), pancreatic cancer (38) and 
hepatocellular carcinoma (39). In addition, miR-10b expression levels were revealed to be associated with clinicopathological factors. In gastric cancer, miR-10b expression levels were associated with the size of tumor, Lauren classification, depth of invasion, lymph node and distant metastasis, TNM stage and prognosis (40). Zhang et al (41) reported that the relative expression levels of miR-10b in non-small-cell lung cancer were significantly positively associated with TNM stage and regional lymph node involvement. Kaplan-Meier analysis revealed that patients with increased levels of miR-10b had significantly poorer survival rate than those with lower expression of miR-10b. Nishida et al (42) revealed that high level miR-10b were associated with high incidence of lymphatic invasion and poor prognosis in patients with colorectal cancer. These studies indicated that miR-10b may be a prognostic target for cancers.

Functionally, miR-10b was validated as an oncogene $(31,32)$. Previous studies demonstrated that ectopic miR-10b expression improved migration and invasion abilities of bladder cancer, nasopharyngeal carcinoma and gastric cancer cells $(31,35,37)$. Liao et al revealed that restoration of miR-10b expression enhanced proliferation, migration and invasion of hepatocellular carcinoma $(39,43)$. In non-small-cell lung cancer, miR-10b overexpression prompted cell proliferation and invasion, and inhibited apoptosis $(32,44)$. However, the expression and functions of miRNAs are tissue specific. miR-10b was reported to be downregulated in clear-cell renal cell carcinoma (45) and was associated with metastasis and progression of clear-cell renal cell carcinoma (46). Increased miR-10b expression inhibited cell proliferation, migration and invasion of clear-cell renal cell carcinoma (45). In the present study, it was verified that miR-10b was downregulated in cervical cancer, and acted as a tumor suppressor. These contradictory results may be explained by the imperfect complementarity of the interactions between miRNAs and target genes (47).

Different cancers have different target genes of miR-10b, including Hoxd10 (31) in gastric cancer, E-cadherin (41) and Krüppel-like factor 4 (44) in non-small-cell lung cancer, ras homolog family member C (RhoC) (34) in colorectal cancer and $\mathrm{RhoC}$, urokinase-type plasminogen activator receptor matrix metalloproteinases (39) and cell adhesion molecule 1 (43) in hepatocellular carcinoma. In the present study, IGF-1R was identified as a novel target gene of miR-10b in cervical cancer. Of numerous potential target genes for miR-10b, predicted by bioinformatics analysis, IGF-1R was selected for the present study. IGF-1R was previously revealed to be upregulated in cervical cancer (48). Luciferase report assay results indicated that miR-10b may directly target the 3'UTR of IGF-1R. In vitro miR-10b overexpression significantly decreased IGF-1R expression, whereas inhibition of miR-10b resulted in increased IGF-1R. In addition, IGF-1R siRNA may mimic the effects of miR-10b overexpression on proliferation, migration and invasion of cervical cancer cells. Thus, the present results suggested that miR-10b acted as a tumor suppressor in cervical cancer, at least in part, through negative regulation of IGF-1R.

In conclusion, to the best of our knowledge, this is the first study to demonstrate that miR-10b is downregulated in cervical cancer. miR-10b acted as a tumor suppressor in cervical cancer by inhibiting cell proliferation, migration and invasion, and directly regulating IGF-1R expression via binding to its 3'UTR. This provides new insights into the mechanisms of initiation and progression of cervical cancer.

\section{References}

1. Jemal A, Bray F, Center MM, Ferlay J, Ward E and Forman D: Global cancer statistics. CA Cancer J Clin 61: 69-90, 2011.

2. Howell LP, Zhou H, Wu W and Davis R: Significance of subclassifying high-grade squamous intraepithelial lesions into moderate dysplasia/CIN II versus severe dysplasia/CIN III/CIS in the bethesda system terminology. Diagn Cytopathol 30: 362-366, 2004.

3. Zeng K, Zheng W, Mo X, Liu F, Li M, Liu Z, Zhang W and Hu X: Dysregulated microRNAs involved in the progression of cervical neoplasm. Arch Gynecol Obstet 292: 905-913, 2015.

4. Wardak S: Human Papillomavirus (HPV) and cervical cancer. Med Dosw Mikrobiol 68: 73-84, 2016.

5. Syrjänen KJ and Syrjänen SM: Human papillomavirus (HPV) typing as an adjunct to cervical cancer screening. Cytopathology 10: 8-15, 1999.

6. Wen Y, Pan XF, Zhao ZM, Chen F, Fu CJ, Li SQ, Zhao Y, Chang H, Xue QP and Yang CX: Knowledge of human papillomavirus (HPV) infection, cervical cancer, and HPV vaccine and its correlates among medical students in Southwest China: A multi-center cross-sectional survey. Asian Pac J Cancer Prev 15: 5773-5779, 2014.

7. Hildesheim $A$ and Wang SS: Host and viral genetics and risk of cervical cancer: A review. Virus Res 89: 229-240, 2002.

8. Martin CM, Astbury K and O'Leary JJ: Molecular profiling of cervical neoplasia. Expert Rev Mol Diagn 6: 217-229, 2006.

9. Bertelsen BI, Steine SJ, Sandvei R, Molven A and Laerum OD: Molecular analysis of the PI3K-AKT pathway in uterine cervical neoplasia: Frequent PIK3CA amplification and AKT phosphorylation. Int J Cancer 118: 1877-1883, 2006.

10. Cheung TH, Lo KW, Yim SF, Chan LK, Heung MS, Chan CS, Cheung AY, Chung TK and Wong YF: Epigenetic and genetic alternation of PTEN in cervical neoplasm. Gynecol Oncol 93: 621-627, 2004.

11. Wang PH, Yang SF, Chen GD, Han CP, Chen SC, Lin LY and Ko JL: Human nonmetastatic clone 23 type 1 gene suppresses migration of cervical cancer cells and enhances the migration inhibition of fungal immunomodulatory protein from Ganoderma tsugae. Reprod Sci 14: 475-485, 2007.

12. Pareja R, Rendón GJ, Sanz-Lomana CM, Monzón O and Ramirez PT: Surgical, oncological, and obstetrical outcomes after abdominal radical trachelectomy-a systematic literature review. Gynecol Oncol 131: 77-82, 2013.

13. Kogo R, How C, Chaudary N, Bruce J, Shi W, Hill RP, Zahedi P, Yip KW and Liu FF: The microRNA-218 Survivin axis regulates migration, invasion and lymph node metastasis in cervical cancer. Oncotarget 6: 1090-1100, 2015.

14. Sevignani C, Calin GA, Siracusa LD and Croce CM: Mammalian microRNAs: A small world for fine-tuning gene expression. Mamm Genome 17: 189-202, 2006.

15. Zhang B, Wang Q and Pan X: MicroRNAs and their regulatory roles in animals and plants. J Cell Physiol 210: 279-289, 2007.

16. Fei B and $\mathrm{Wu} \mathrm{H}: \mathrm{MiR}-378$ inhibits progression of human gastric cancer MGC-803 cells by targeting MAPK1 in vitro. Oncol Res 20: 557-564, 2012.

17. Wang Z, Yin B, Wang B, Ma Z, Liu W and Lv G: MicroRNA-210 promotes proliferation and invasion of peripheral nerve sheath tumor cells targeting EFNA3. Oncol Res 21: 145-154, 2013.

18. Zhang WH, Gui JH, Wang CZ, Chang Q, Xu SP, Cai CH, Li YN, Tian YP, Yan L and Wu B: The identification of miR-375 as a potential biomarker in distal gastric adenocarcinoma. Oncol Res 20: 139-147, 2012.

19. Misawa A, Katayama R, Koike S, Tomida A, Watanabe T and Fujita N: AP-1-Dependent miR-21 expression contributes to chemoresistance in cancer stem cell-like SP cells. Oncol Res 19: 23-33, 2010.

20. Ohdaira H, Sekiguchi M, Miyata K and Yoshida K: MicroRNA-494 suppresses cell proliferation and induces senescence in A549 lung cancer cells. Cell Prolif 45: 32-38, 2012.

21. Cho WC, Chow AS and Au JS: Restoration of tumour suppressor hsa-miR-145 inhibits cancer cell growth in lung adenocarcinoma patients with epidermal growth factor receptor mutation. Eur J Cancer 45: 2197-2206, 2009. 
22. Liu T, Tang H, Lang Y, Liu M and Li X: MicroRNA-27a functions as an oncogene in gastric adenocarcinoma by targeting prohibitin. Cancer Lett 273: 233-242, 2009.

23. Wu D, Zhou Y, Pan H, Zhou J, Fan Y and Qu P: microRNA-99a inhibiting cell proliferation, migration and invasion by targeting fibroblast growth factor receptor 3 in bladder cancer. Oncol Lett 7: 1219-1224, 2014.

24. Li E, Zhang J, Yuan T and Ma B: MiR-145 inhibits osteosarcoma cells proliferation and invasion by targeting ROCK1. Tumour Biol 35: 7645-7650, 2014

25. Gururajan M, Josson S, Chu GC, Lu CL, Lu YT, Haga CL, Zhau HE, Liu C, Lichterman J, Duan P, et al: miR-154* and miR-379 in the DLK1-DIO3 microRNA mega-cluster regulate epithelial to mesenchymal transition and bone metastasis of prostate cancer. Clin Cancer Res 20: 6559-6569, 2014

26. Wang Z, Ma X, Cai Q, Wang X, Yu B, Cai Q, liu B, Zhu Z and Li C: MiR-199a-3p promotes gastric cancer progression by targeting ZHX1. FEBS Lett 588: 4504-4512, 2014.

27. Liu T, Hou L and Huang Y: EZH2-specific microRNA-98 inhibits human ovarian cancer stem cell proliferation via regulating the pRb-E2F pathway. Tumour Biol 35: 7239-7247, 2014.

28. Qiu F, Sun R, Deng N, Guo T, Cao Y, Yu Y, Wang X, Zou B, Zhang S, Jing T, et al: miR-29a/b enhances cell migration and invasion in nasopharyngeal carcinoma progression by regulating SPARC and COL3A1 gene expression. PLoS One 10: e0120969, 2015.

29. Alizadeh S, Kaviani S, Soleimani M, Abroun S, Kashani-KhatibZ, Asgharzadeh A, Dargahi H and Mousavi R: Mir-55 inhibition can reduce cell proliferation and induce apoptosis in Jurkat (Acute T cell Leukemia) cell line. Iran J Ped Hematol Oncol 4: 141-150, 2014

30. Saldanha G, Elshaw S, Sachs P, Alharbi H, Shah P, Jothi A and Pringle JH: microRNA-10b is a prognostic biomarker for melanoma. Mod Pathol 29: 112-121, 2016.

31. Wang YY, Li L, Ye ZY, Zhao ZS and Yan ZL: MicroRNA-10b promotes migration and invasion through Hoxd10 in human gastric cancer. World J Surg Oncol 13: 259, 2015.

32. Huang J, Sun C, Wang S, He Q and Li D: microRNA miR-10b inhibition reduces cell proliferation and promotes apoptosis in non-small cell lung cancer (NSCLC) cells. Mol Biosyst 11: 2051-2059, 2015

33. Ji Y, Wei Y, Wang J, Gong K, Zhang Y and Zuo H: Correlation of microRNA-10b upregulation and poor prognosis in human gliomas. Tumour Biol 36: 6249-6254, 2015.

34. Wang YF, Li Z, Zhao XH, Zuo XM, Zhang Y, Xiao YH, Li J and Peng ZH: MicroRNA-10b is upregulated and has an invasive role in colorectal cancer through enhanced Rhoc expression. Oncol Rep 33: 1275-1283, 2015.

35. Xiao H, Li H, Yu G, Xiao W, Hu J, Tang K, Zeng J, He W, Zeng $\mathrm{G}$, Ye Z and Xu H: MicroRNA-10b promotes migration and invasion through KLF4 and HOXD10 in human bladder cancer. Oncol Rep 31: 1832-1838, 2014.
36. Han X, Yan S, Weijie Z, Feng W, Liuxing W, Mengquan L and Qingxia F: Critical role of miR-10b in transforming growth factor- $\beta 1$-induced epithelial-mesenchymal transition in breast cancer. Cancer Gene Ther 21: 60-67, 2014.

37. Sun XJ, Liu H, Zhang P, Zhang XD, Jiang ZW and Jiang CC miR-10b promotes migration and invasion in nasopharyngeal carcinoma cells. Asian Pac J Cancer Prev 14: 5533-5537, 2013.

38. Ouyang H, Gore J, Deitz S and Korc M: microRNA-10b enhances pancreatic cancer cell invasion by suppressing TIP30 expression and promoting EGF and TGF- $\beta$ actions. Oncogene 33: 4664-4674, 2014.

39. Liao CG, Kong LM, Zhou P, Yang XL, Huang JG, Zhang HL and Lu N: miR-10b is overexpressed in hepatocellular carcinoma and promotes cell proliferation, migration and invasion through RhoC, uPAR and MMPs. J Transl Med 12: 234, 2014.

40. Wang YY, Ye ZY, Zhao ZS, Li L, Wang YX, Tao HQ, Wang HJ and He XJ: Clinicopathologic significance of miR-10b expression in gastric carcinoma. Hum Pathol 44: 1278-1285, 2013.

41. Zhang J, Xu L, Yang Z, Lu H, Hu D, Li W, Zhang Z, Liu B and Ma S: MicroRNA-10b indicates a poor prognosis of non-small cell lung cancer and targets E-cadherin. Clin Transl Oncol 17: 209-214, 2015.

42. Nishida N, Yamashita S, Mimori K, Sudo T, Tanaka F, Shibata K, Yamamoto H, Ishii H, Doki Y and Mori M: MicroRNA-10b is a prognostic indicator in colorectal cancer and confers resistance to the chemotherapeutic agent 5-fluorouracil in colorectal cancer cells. Ann Surg Oncol 19: 3065-3071, 2012.

43. Li QJ, Zhou L, Yang F, Wang GX, Zheng H, Wang DS, He Y and Dou KF: MicroRNA-10b promotes migration and invasion through CADM1 in human hepatocellular carcinoma cells. Tumour Biol 33: 1455-1465, 2012.

44. Liu Y, Li M, Zhang G and Pang Z: MicroRNA-10b overexpression promotes non-small cell lung cancer cell proliferation and invasion. Eur J Med Res 18: 41, 2013.

45. He C, Zhao X, Jiang H, Zhong Z and Xu R: Demethylation of miR-10b plays a suppressive role in ccRCC cells. Int J Clin Exp Pathol 8: 10595-10604, 2015.

46. Wu X, Weng L, Li X, Guo C, Pal SK, Jin JM, Li Y, Nelson RA, $\mathrm{Mu} \mathrm{B}$, Onami SH, et al: Identification of a 4-microRNA signature for clear cell renal cell carcinoma metastasis and prognosis. PLoS One 7: e35661, 2012.

47. Yu Z, Ni L, Chen D, Zhang Q, Su Z, Wang Y, Yu W, Wu X, Ye J, Yang S, et al: Identification of miR-7 as an oncogene in renal cell carcinoma. J Mol Histol 44: 669-677, 2013.

48. Steller MA, Delgado CH, Bartels CJ, Woodworth CD and Zou Z: Overexpression of the insulin-like growth factor-1 receptor and autocrine stimulation in human cervical cancer cells. Cancer Res 56: 1761-1765, 1996. 